\title{
Present and future trends of biologic therapies and small molecules in hidradenitis suppurativa
}

\author{
Antonio Martorell ${ }^{1 *}$, Abdulhadi Jfri², Gemma Ochando', and Fatima Mayo ${ }^{1,3}$ \\ ${ }^{1}$ Inflammatory Diseases Unit, Department of Dermatology, Venereology and Surgery, Hospital de Manises, Valencia, Spain; ${ }^{2} \mathrm{Harvard}$ Medical School, \\ Boston, United States; ${ }^{3}$ Fundación Instituto Valenciano de Oncología, Valencia, Spain
}

\begin{abstract}
Background: Hidradenitis suppurativa (HS) is a chronic inflammatory skin disease affecting areas with a high density of apocrine glands and characterized by subcutaneous nodules that may evolve into fistulas with pus secretion. Progressor and aggressive profiles will require the use of immunomodulatory therapies to fight against their disease outcomes. Various options have been proposed to treat HS. Unfortunately, no therapy has been fully successful. Methods: The aim of this review is to investigate all current knowledge on biologic and small molecule options for HS management. A systematic literature research using the words "biologic," "small molecule," "therapy," and "HS" was performed in PubMed/Medline and Scopus/ Embase databases. A search of the clinicaltrials.gov website for interventional recruiting and completed trials including the term "HS" was also performed up to August 2021. Results: At present, tumor necrosis factor TNF alfa blockers are considered the first therapeutic option based on clinical trials results and real-world evidence. However, new therapeutic options based on alternative pathway blockage, including interleukin (IL)-17, Complement and IL-23, seem to offer future alternatives for this condition. Conclusions: Several future studies and clinical trials are necessary to gain new knowledge about HS and to properly treat this complex condition. At present, IL-17 blockers such as secukinumab and bimekizumab represent the most promising alternative therapies for those patients who do not respond to adalimumab.
\end{abstract}

Keywords: Hidradenitis suppurativa. Biologic. Small molecule. Therapy. Clinical trials.

\section{Introduction}

Hidradenitis suppurativa (HS) is a chronic inflammatory condition primarily affecting apocrine-gland-rich regions of the body such as the axillary and groin areas $^{1}$. HS presents with painful nodules and abscesses that may coalesce and form fistulas with pus drainage.

Lesions often evolve into scars, with patients suffering significant physical and psychological impact ${ }^{2}$. Various therapies have been proposed to treat HS. Unfortunately, no therapy has been fully successful in the control of this disease ${ }^{3}$.

At present, HS management is evolving to a more integral intervention in which a holistic evaluation of dynamic inflammatory lesions, static non-reversible structures and their related comorbidities will be mandatory to achieve the best results for patients (Fig. 1) ${ }^{2}$.

One of the most important challenges in managing $\mathrm{HS}$ is to better understand the disease history to stop possible progression to irreversible lesions.

The window of opportunity for HS treatment is the period during which efforts to control inflammatory activity will be most effective. This period occurs in the early stages of the disease, before the onset of sequelae and established irreversible damage. Immunomodulatory therapy during this phase may alter the natural history of the disease by reducing the accumulation of tissue damage (Fig. 2).
Correspondence:

Received: 13-09-2021

*Antonio Martorell

Accepted: 25-10-2021

DOI: 10.24875/JIMIDS.M21000012

E-mail: martorelldermatologia @gmail.com
Available online: 25-01-2022 J IMIDs. 2022;2(1):8-19

www.JournalofIMIDs.com 


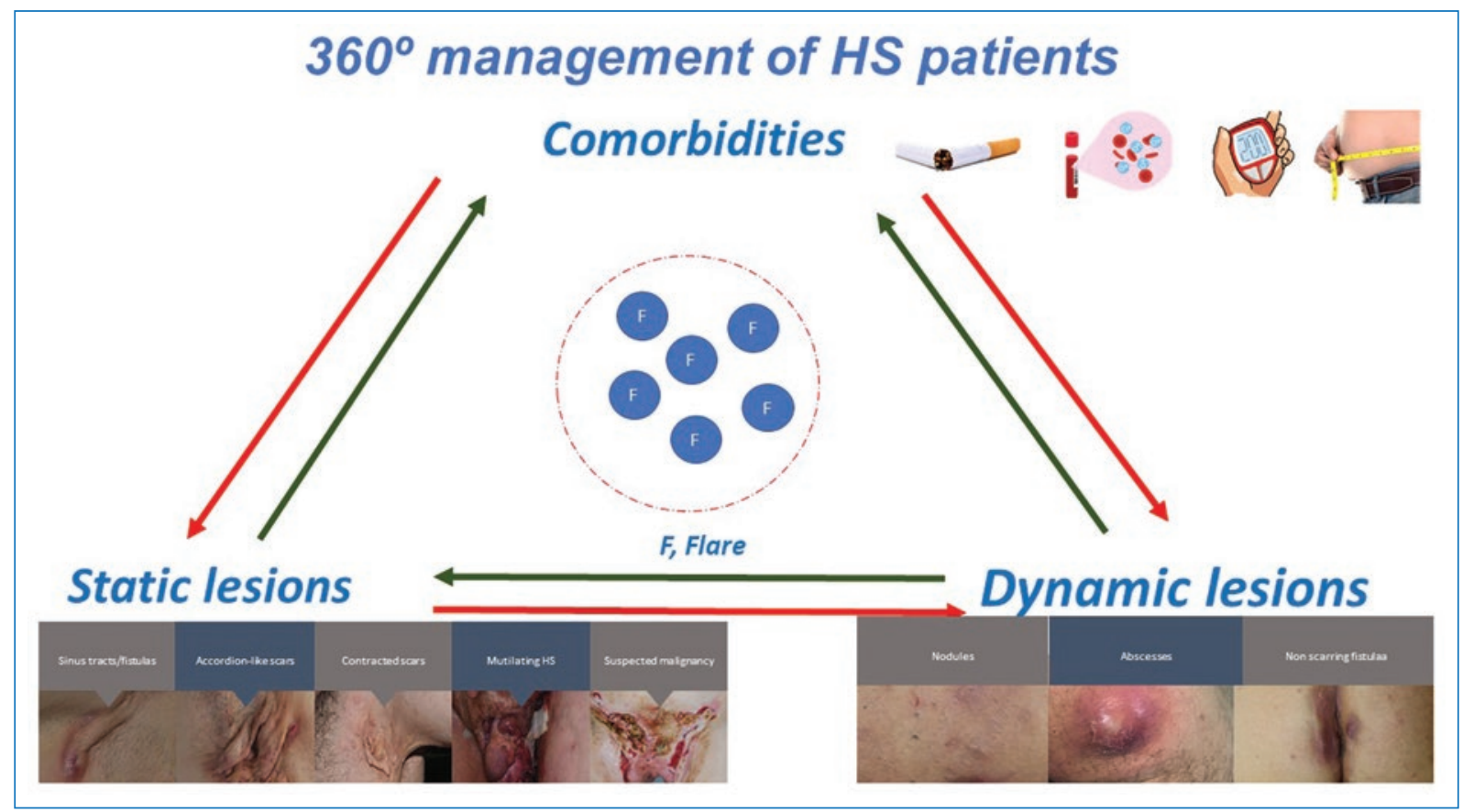

Figure 1. $360^{\circ}$ Management of HS patients.

\section{The nature of HS}

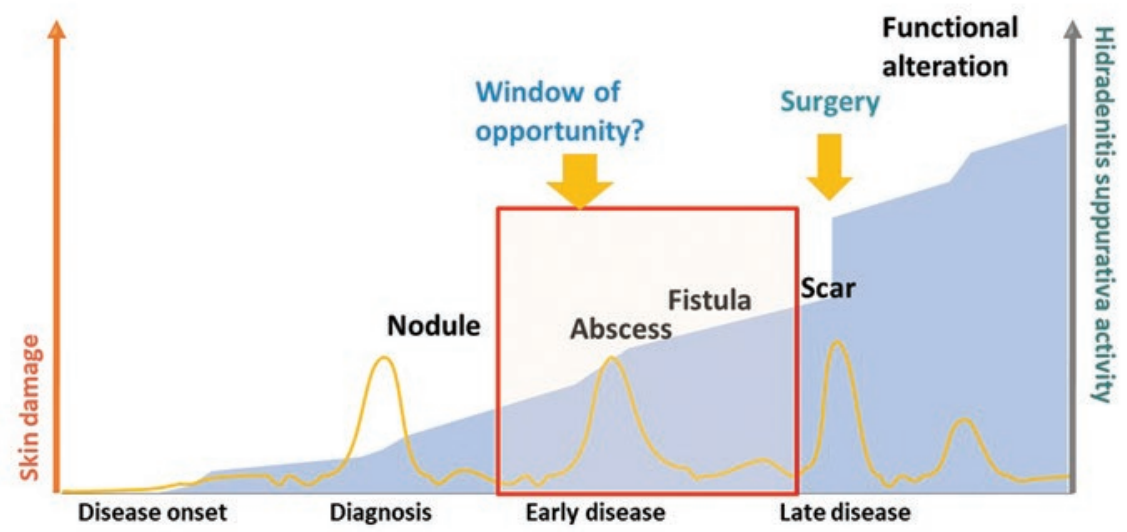

Figure 2. Window of opportunity in HS (adapted from Martorell et al. 2016 ${ }^{4}$ ).

Disease progression occurs in up to $30 \%$ of HS patients, so the early detection of aggressive cases is critical to changing the course of disease in these patients.

Recently, Martorell et al. ${ }^{3}$ conducted an observational, descriptive, nonrandomized, prospective study with
197 patients and described a clinical phenotype referred to as "inflammatory" that showed an independent risk for disease aggressiveness in the multivariate analysis (odds ratio [OR] 0.034 [95\% Confidence interval: 0.015, 0.072]) (Table 1 and Fig. 3). Early detection of these 
Table 1. Clinical features of HS phenotypes

\begin{tabular}{|c|c|}
\hline Follicular phenotype & Inflammatory phenotype \\
\hline - Females $>$ males & - Males > Females \\
\hline - Frequent HS family history & - Rare HS family history \\
\hline - Peripuberal onset (mean, 14-18 years) & $\begin{array}{l}\text { - Adolescent and adult onset ( } 18-25 \text { or }>35 \text { years) } \\
\text { - Rare child cases (onset, 8-12 years) }\end{array}$ \\
\hline - Typical sites: thighs/axillar folds & - Typical sites: inguinal/axillar folds and buttocks \\
\hline - Presence of folliculitis and/or comedones & $\begin{array}{l}\text { - Folliculitis/comedones, epidermal cysts and nodules } \\
\text { are scarce }\end{array}$ \\
\hline - The main active lesion is the nodule & $\begin{array}{l}\text { - Main active lesions are abscesses and wide tunnels } \\
\text { and fibrosing tracts }\end{array}$ \\
\hline - Individual predisposition to develop epidermal cysts & $\begin{array}{l}\text { - Tendency to confluence in poorly defined } \\
\text { inflammatory and scarring plaques }\end{array}$ \\
\hline - Post-inflammatory pigmentation may occur & $\begin{array}{l}\text { MIXED PHENOTYPE } \\
\text { FIllicular progression to } \\
\text { Inflammatory (heavy smokers, } \\
\text { combined commedons and } \\
\text { abscesses/tunnels) }\end{array}$ \\
\hline - Abscesses and tunnels are rare, the latter being thin and non-branching & $\begin{array}{l}\text { - Subcutaneous or interfacial penetrating fistulas can } \\
\text { be detected }\end{array}$ \\
\hline - Non-progressive behavior & - Progressive/aggressive behavior \\
\hline
\end{tabular}

HS: hidradenitis suppurativa; y: year. Adapted from Martorell, et al. (2020)

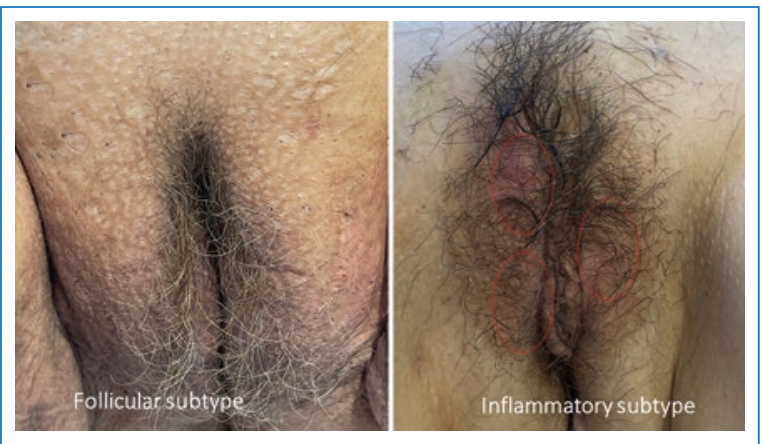

Figure 3. Follicular subtype and Inflammatory subtype in HS.

progressive profiles will require the use of immunomodulatory therapies to address this outcome (Fig. 4)2.

Various therapies have been proposed to treat HS. Unfortunately, no therapy has been fully successful at controlling the disease ${ }^{1-3}$. Among immunodulatory therapies, multiple biologic therapies and small molecules have been suggested.

During the present review we will analyze the real evidence of current HS options and future options that

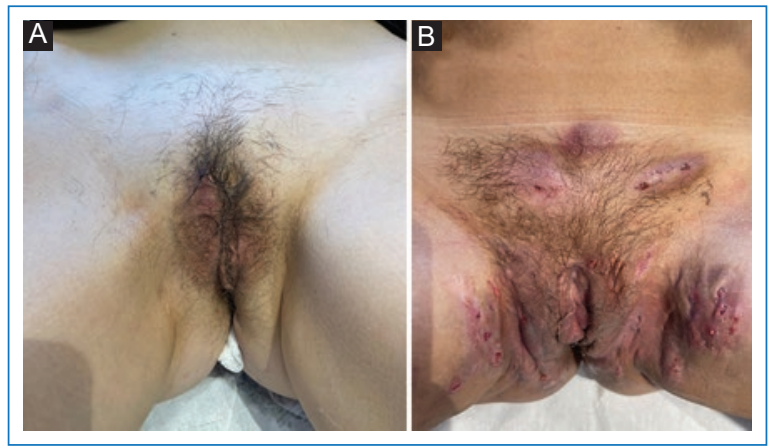

Figure 4. Progression of the disease. A: Month 0; B: Month 12.

\section{Material and methods}

A systematic literature research was performed in PubMed/Medline, Scopus/Embase, and Google Scholar, to find articles relevant to this review. Keywords searched included "biologic," "small molecule," "therapy," and "HS." Duplicate articles were deleted and articles that introduced no new information were excluded from the study. A search of the website clinicaltrials.gov for interventional recruiting and completed clinical trials with the term "HS" through August 31, 2021 was also conducted. 


\section{OVERVIEW OF TARGETS IN DEVELOPMENT}
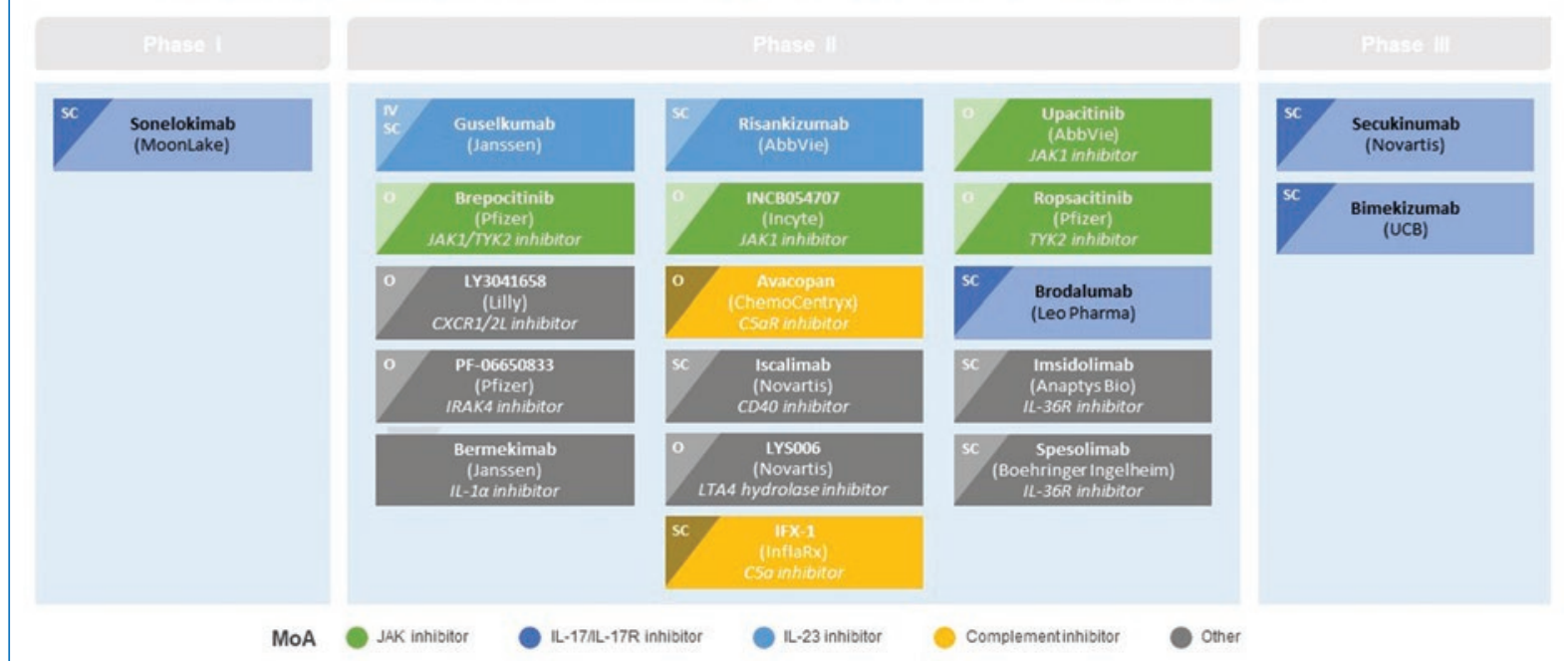

Figure 5. Pipeline of biologics and small molecules in HS (adapted from Clinicaltrials.gov) ${ }^{5}$.

CD: cluster of differentiation; CXCR: C-X-C chemokine receptor; IFX: infliximab; IL: interleukin; IRAK4: interleukin-1 receptor-associated kinase 4; IV: intravenous; JAK: Janus kinase; LTA4: leukotriene A4; MoA: mechanism of action; O: oral; R: receptor; SC: subcutaneous; TYK2: tyrosine kinase 2.

\section{Results}

\section{New data from current approved biologic therapy in $\mathrm{HS}$}

\section{Adalimumab}

Adalimumab is the only currently-approved biologic for the treatment of moderate-to-severe HS in several countries worldwide ${ }^{3,6}$. Its inhibitory action prevents the movement of nuclear transcription factor nuclear factor kappa B into the nucleus, where it induces the production of cytokines that contribute to the inflammatory cascade $^{6}$. It also downregulates processes induced or regulated by tumor necrosis factor (TNF) as inhibiting endothelial-leukocyte adhesion molecule-1, intracellular adhesion molecule-1, and vascular cell adhesion molecule-1. All of these molecules are responsible for the migration of leukocytes ${ }^{6}$.

Two studies, PIONEER I and II, had a primary endpoint of achieving HS clinical response (HiSCR). This was defined as $a \geq 50 \%$ reduction from baseline in total abscess and inflammatory nodule count (AN count) without increases in abscess or draining fistula counts relative to baseline. Each of the two was a similarly designed, randomized Phase III clinical trial, and both trials demonstrated that a significantly greater percentage of patients achieved HiSCR at week 12 with adalimumab than with placebo: $41.8 \%$ versus $26.0 \%$ in
PIONEER I ( $p=0.003$ ) and $58.9 \%$ versus $27.6 \%$ in PIONEER II $(p<0.001)$. They also both showed that adalimumab significantly improved health-related quality of life?

Adults and adolescents weighing $60 \mathrm{~kg}$ and greater should be dosed at a level of $160 \mathrm{mg}$ of adalimumab on day 1 followed by $80 \mathrm{mg}$ on day 15 . Following this the dosage can be dropped to $40 \mathrm{mg}$ at day 29 and every week thereafter. Treatment with adalimumab should be reconsidered for patients that show no improvement by week 12, and during treatment daily use of a topical antiseptic wash on HS lesions is recommended?.

Recently, Marzano et al. published their real-world experience with adalimumab in $\mathrm{HS}$ patients ${ }^{8}$. Preliminary conclusions included the fact that a window of opportunity, as previously proposed by Martorell, could exist due to the fact that administration of adalimumab in early phases improves the results achieved on HS patients in terms of HiSCR. These results, which encourage the use of biologics from the time of symptom onset in the most inflammatory forms of HS (such as the inflammatory or mixed phenotypes) (Table 1 and Figs. 2 and 3 ) to halt the disease progression (Fig. 4A and B), contradicts the labeling of adalimumab as a second-line therapy after failure to respond to conventional systemic treatments (e.g., systemic antibiotics $)^{9}$. 
Adalimumab's safety profile in patients with HS is consistent with the known adverse effect profile of the drug, with the most common effects including infections (e.g., upper respiratory, sinusitis), injection site reactions, headache, and rash. The drug is linked to an increased risk of developing serious infections and may have an increased risk of developing malignancies. In the United States the drug carries a black box warning about the risk of serious infections (e.g., bacterial sepsis, invasive fungal infections, tuberculosis, infections due to other opportunistic pathogens), and malignancy (e.g., lymphomas in children and adolescents) ${ }^{6}$. Still, a recent assessment of the risk of adalimumab use for HS during the COVID-19 pandemic supported the use of adalimumab in otherwise healthy patients with HS as long as they were without risk factors, indicating that its use should not predispose them to infection or nasopharyngitis ${ }^{10}$.

\section{Data from off-label options used in the current clinical practice}

\section{Infliximab (IFX)}

IFX has been used as an off-label treatment in patients whose HS is resistant to adalimumab. It is currently FDA-approved for use in inflammatory bowel disease, rheumatoid arthritis, ankylosing spondylitis, psoriatic arthritis, and plaque psoriasis ${ }^{11}$. It acts by binding and blocking both membrane-bound and soluble TNF-alfa that play a central role in the pathologic inflammatory response associated with $\mathrm{HS}^{12}$.

A Phase II double-blind placebo-controlled randomized trial of IFX in moderate-to-severe HS demonstrated a $50 \%$ or greater decrease from baseline HS Severity Index score as well as a statistically and clinically significant improvement from baseline. This improvement was observed at week 8 using the Dermatology Life Quality Index (DLQI) score, visual analog scale (VAS) score, erythrocyte sedimentation rate, and C-reactive protein compared with placebo ${ }^{12}$.

Dosage of IFX is established at $5 \mathrm{mg} / \mathrm{kg}$ body weight on weeks 0,2 , and 6 and thereafter every 8 weeks as an intravenous infusion. However, reports suggest that the dosing regimen for HS requires further refinement, and the North American Clinical HS Management Guidelines recently emphasized the need for dose-ranging studies to optimize management ${ }^{13}$. Along these lines, Ghias et al. performed a prospective analysis of 42 patients initiating IFX $7.5 \mathrm{mg} / \mathrm{kg}$ every 4 weeks (IFX 7.5) and 16 patients receiving dose escalation to
IFX $10 \mathrm{mg} / \mathrm{kg}$ every 4 weeks (IFX 10) between March 1 , 2018, and February 28, 2019. The primary outcome measure (clinical response) was the proportion of patients with Physician Global Assessment (PGA) of clear, minimal, or mild (score of 0-2) HS with at least a 2-grade improvement from baseline scores. In the result analysis, the proportion of patients achieving a clinical response after initiating IFX 7.5 was 20 of $42(47.6 \%)$ at week 4 and 17 of $24(70.8 \%)$ at week 12. For patients receiving dose escalation to IFX 10 because of incomplete initial response, 6 of 16 (37.5\%) achieved clinical response at week 4 and 6 of $12(50 \%)$ at week 12. The main conclusion was that the initiation of IFX 7.5 every 4 weeks, with possible dose escalation to IFX 10 if needed, provides optimal mitigation of HS-related disease activity ${ }^{14}$.

Herpes simplex infection and influenza-like illness were the most common adverse effects in the Phase II clinical trial. IFX has also been associated with paradoxical worsening of facial acne vulgaris, demyelinating neuropathies, and metastatic cutaneous squamous cell carcinoma in some cases, as well as a case of Gemella morbillorum bacteremia complicated by brain abscesses $^{13}$.

\section{Anakinra}

Anakinra is a recombinant interleukin (IL)-1 receptor inhibitor that competitively inhibits the binding of both IL- $1 \alpha$ and IL-1 $\beta$ to the IL-1 type 1 receptor. It has been used off-label for managing HS, but further and higher-quality data is needed to clarify its therapeutic role. It is currently FDA-approved for use in rheumatoid arthritis and neonatal-onset multisystem inflammatory disease ${ }^{15}$.

IL 1 is produced in response to various microbial and non-microbial stimuli and plays a key role in immune dysregulation in $\mathrm{HS}^{12}$. A double-blind placebo-controlled randomized clinical trial included 20 patients with moderate-to-severe HS (Hurley II or III), and demonstrated that the use of anakinra created a significant statistical difference in the primary outcome, including a decrease in DLQI scores from weeks 0 to 12. Still, between weeks 12 and 24 there was no significant overall difference in DLQI between the study arms. The secondary endpoint showed anakinra delivered a significantly increased prolongation to new HS exacerbation ${ }^{15}$. Another open-label trial of anakinra revealed that at week 8 there was a significant and clinically meaningful mean decrease in Sartorius score (a validated scoring system for symptom severity in HS) 
and DLQI scores, but mean Sartorius scores rebounded to baseline for 4-out-of-5 patients by week 16 and so did mean DLQI scores ${ }^{16}$.

Dosing of anakinra is $100 \mathrm{mg}$ subcutaneous daily dose. The most reported adverse effect is a painful injection site reaction, though there are case reports indicating failure of anakinra therapy in HS, or even a worsening of HS, with report of drug-induced sarcoidosis in HS patients ${ }^{17}$.

\section{Ustekinumab}

Ustekinumab is a human monoclonal antibody that acts by binding to and inhibiting the p40 subunit on IL-12 and IL-23 which may be elevated in HS lesions ${ }^{12}$. It is FDA-approved for use in plaque psoriasis, psoriatic arthritis, and Crohn's disease and has been used off-label for managing $\mathrm{HS}^{18}$.

A case series of patients administered ustekinumab demonstrated an improvement in the PGA score in $70 \%$ (7/10) of participants and an improvement in the Numerical Pain Rating Scale in $80 \%(8 / 10)^{13}$. A systematic review of ustekinumab reported that $76 \%(34 / 45)$ of patients experienced clinical improvement in disease severity, and $84 \%$ (38/45) experienced symptomatic improvement ${ }^{19}$.

Dosing for patients weighing $100 \mathrm{~kg}$ and below is $45 \mathrm{mg}$ per dose, while those weighing above $100 \mathrm{~kg}$ received a dose of $90 \mathrm{mg}$, though the Phase II study's authors suggested that further intensification of the dosing regimen in HS may be necessary ${ }^{19}$.

Real world experience with ustekinumab suggests the need to use it in Crohn's posology with a single intravenous infusion dose of STELARA ${ }^{\circledR}$ using the weight-based dosage regimen followed by a subcutaneous regimen with a recommended maintenance dosage of $90 \mathrm{mg} 8$ weeks after the initial intravenous dose, then every 8 weeks thereafter to achieve better results in HS patients ${ }^{20}$.

The systematic review reported headache, fatigue, and upper respiratory tract infection as the most frequently reported adverse events, none of which were severe. All spontaneously resolved and the case series reported observing no adverse events related to ustekinumab use ${ }^{21}$.

\section{Apremilast}

Apremilast is a phosphodiesterase 4 inhibitor that exerts an immunomodulatory action. It partially blocks the expression of pro-inflammatory cytokines and induces the expression of anti-inflammatory cytokines. The drug has been used off-label for managing $\mathrm{HS}^{22}$ and is currently indicated in adult patients diagnosed with plaque psoriasis and/or psoriatic arthritis who do not respond to conventional systemic therapy.

Apremilast acts on T cells, natural killer cells, neutrophils, monocytes, dendritic cells, and various cells involved in the pathogenesis of $\mathrm{HS}^{23}$. A double-blind, randomized clinical trial showed that 8-out-of-15 patients with moderate HS (53.3\%) achieved a positive HiSCR at week 16 after being given apremilast compared to zero of five in the placebo group. These patients also showed a significantly lower abscess and nodule count and lower numerical rating scales for pain with no significant difference between both arms in the DLQI over the period of the trial24. A case series of nine patients (with three patients dropping out) with moderate-to-severe HS treated with apremilast for 5-9 months showed that 5 out of 6 patients reported achieving significant improvement in terms of Sartorius score, pain, and in the DLQI scores $^{24}$. An open-label, phase 2 clinical trial that enrolled 20 patients with mild-to-moderate HS revealed that $65 \%$ of patients achieved HiSCR30 at weeks 16 and week 24, with patients reporting significant mean improvements in the overall Sartorius score, the PGA score, the VAS pain score, and the DLQI score ${ }^{24}$.

Dosage of apremilast is either $30 \mathrm{mg}$ twice daily or $30 \mathrm{mg}$ once daily as long as creatinine clearance is $>30 \mathrm{ml} / \mathrm{min}^{24}$. There were mild-to-moderate headache and gastrointestinal symptoms (diarrhea and nausea) reported by patients in the randomized clinical trial, as well as depression ${ }^{24}$.

\section{Ongoing clinical trials in HS}

\section{Phase I drugs}

\section{Sonelokimab (Moonlake)}

Sonelokimab (also known as M1095) is a novel trivalent nanobody comprised of monovalent camelid-derived (i.e., from the Camelidae family of mammals, such as camels, llamas, and alpacas) nanobodies specific to human IL-17A, IL-17F, and human serum albumin. Nanobodies are a novel class of proprietary therapeutic proteins based on single-domain, camelid and heavychain-only antibodies.

Its smaller size may allow them to penetrate deeper into solid tissues such as skin and joints. In addition, their high stability, strong antigen-binding affinity, water solubility and natural origin make them suitable for development into next-generation biodrugs ${ }^{25}$. 
This molecule is now being tested for different inflammatory diseases such as psoriasis, psoriatic arthritis, ankylosing spondylitis, and HS.

Phase II, multicenter, randomized, and placebo-controlled trials have already been published for psoriasis. Under this indication, sonelokimab in doses of $120 \mathrm{mg}$ or less showed significant clinical benefit over placebo, with rapid onset of treatment effect, durable improvements, and an acceptable safety profile. Nasopharyngitis and pruritus are the most frequent adverse events ${ }^{26}$.

\section{Phase II drugs}

\section{LY3041658 (LILLY) CXCR1/2L INHIBITOR}

CXC chemokines and their receptors play an important role in increasing inflammation in HS by attracting neutrophils, dendritic cells and memory $T$ and $B$ cells attractants ${ }^{27}$.

LY3041658 is a humanized monoclonal antibody that blocks CXCR1 and CXCR2 signaling ${ }^{28}$. A multicenter, randomized, double-blind, placebo-controlled, Phase 2 study is underway to evaluate the efficacy and safety of LY3041658 in adults with moderate-to-severe HS $^{29}$. Currently the recruitment phase is still open, thus data about the drug efficacy and safety are not yet available ${ }^{29}$.

\section{C5AR INHIBITORS}

Selectively blocking the action of $\mathrm{C} 5 \mathrm{a}$ on its receptor is crucial to reducing the risk of impairing the formation of the membrane attack complex C5b-9 necessary for defense against encapsulated bacterial infections, including Neisseria meningitidis ${ }^{30}$.

HS is characterized by inflammation and an abundant neutrophilic infiltrate. It has been described as a complementary system that plays a fundamental role in the infiltration and activation of neutrophils in the skin ${ }^{31,32}$.

It has recently been shown that circulating $\mathrm{C} 5 \mathrm{a}$ and C5b5-9 are increased in HS, and consumption of circulating $\mathrm{C} 5 \mathrm{a}$ is associated with disease severity. Moreover, C5a primes the overproduction of TNF-alpha by circulating mononuclear cells ${ }^{31}$.

Avacopan and vilobelimab are the two C5aR inhibitors that are running clinical assays.

\section{*Avacopan (ChemoCentryx)}

*Avacopan (CCX168) is an orally administered selective and potent $\mathrm{C} 5 \mathrm{aR}$ inhibitor ${ }^{33}$.
A randomized, double-blind, placebo-controlled, and parallel group Phase 2 study of avacopan in subjects with moderate (Hurley Stage II) to severe HS (Hurley Stage III) began in December 2018.

The multicenter study randomized 398 patients to three treatment groups. One group was treated with $10 \mathrm{mg}$ avacopan twice daily. The second group was treated with $30 \mathrm{mg}$ avacopan twice daily, and the third group with placebo twice daily for 12 weeks. Primary efficacy analysis took place at 12 weeks ${ }^{30}$. Avacopan $30 \mathrm{mg}$ twice daily demonstrated a significant improvement compared to placebo in Hurley Stage III patients (42.6\% vs. $22.2 \%$, respectively).

The sponsor company plans to advance the drug into Phase III clinical trial for the treatment of severe $\mathrm{HS}^{33}$.

Avacopan demonstrated a favorable safety profile, with fewer adverse events than placebo $(48.5 \%$ vs. $55 \%$, respectively) $)^{34}$.

In addition, after the first 12 weeks, subjects on placebo will be re-randomized $1: 1$ to receive $10 \mathrm{mg}$ or $30 \mathrm{mg}$ of avacopan twice daily for an additional 24 weeks. Subjects treated with avacopan will continue to receive the same dose during the additional 24 weeks. The subjects will be followed for 44 weeks for assessment of safety and efficacy ${ }^{30}$.

\section{*IFX-1 (Infla-Rx) C5aR inhibitor}

*IFX-1 (vilobelimab) is an intravenous monoclonal IgG4 kappa antibody that selectively binds to C5a and blocks its biological activity ${ }^{35,36}$.

The prospective, open-label, and single-arm Phase 2a study of IFX-1 in HS showed a treatment response of $75 \%$ at the end of the treatment and $83.3 \%$ at the end of the 3-month follow-up period, with good tolerance and a decrease in fistulization ${ }^{36}$.

A randomized, double-blind, placebo-controlled, and multicenter Phase 2 trial (SHINE) to determine the efficacy and safety of IFX-1 in 179 subjects with moderate-to-severe HS found HiSCR rates of $38.7 \%-51.5 \%$ across four dosing regimens at week 16 . Remarkably, the placebo treated group reached similar response rates $(47.1 \%)^{37}$.

\section{BERMEKIMAB (JANSSEN) IL-1a INHIBITOR}

Bermekimab (MABp1), unlike anakinra, is a selective inhibitor of IL-1 $\alpha$. IL-1 $\alpha$, a highly pro-inflammatory cytokine with increased levels in HS lesions compared with healthy $\operatorname{skin}^{35,38}$. 
Four clinical trials have studied this fully humanized monoclonal antibody. The first clinical trial revealed a statistically significant improvement in patients treated with bermekimab compared to those receiving placebo (60\% vs. $10 \%$; p: 0.035$)$. This result was supported by the open label extension of the study ${ }^{39,40}$. A new Phase $2 \mathrm{a} / 2 \mathrm{~b}$, multicenter, randomized, placebo and active comparator-controlled, double-blind, dose-ranging study with bermekimab in moderate-to-severe HS is currently under way. Its primary outcome is to assess the percentage of patients who achieve HiSCR50 at week $16^{41}$.

\section{IL-36R INHIBITOR}

IL-36 cytokines are part of the IL-1 superfamily ${ }^{35,42}$. Evidence of IL-36 role in HS is increasing, similar to the well-known hyperactivation of the IL-1 pathway that contributes to the immune dysregulation in $\mathrm{HS}^{35}$.

IL-36 cytokines are predominantly expressed in the skin, where the IL-36 receptor (IL-36R) pathway activates dendritic cells, affects neutrophil recruitment, and participates in polarizing Th1 cells and Th17 cells and their cytokines in serum and lesional HS skin ${ }^{35,42}$. At the moment there is no data about the efficacy of IL-36R inhibitors.

Two of such molecules are undergoing Phase 2 study: Imsidolimab (Anaptys Bio) and Spesolimab (Nochringer Ingelheim).

\section{+IMSIDOLIMAB (ANAPtys BIO) IL-36R INHIBITOR}

An ongoing Phase 2 multicenter, randomized, double-blind, placebo-controlled study of imsidolimab with HS patients will assess the efficacy and safety of this subcutaneous anti IL-36R monoclonal antibody. This study will also characterize the pharmacokinetic profile of imsidolimab and explore the immune response to imsidolimab in subjects with $\mathrm{HS}^{43}$.

\section{+Spesolimab (Nochringer INGelheim) IL-36R INHIBITOR}

Spesolimab is a subcutaneous IL-36R inhibitor that is currently being evaluated in two HS Phase 2 studies. The first study intends to evaluate the evolution of the disease considering the total abscess and inflammatory nodule count after 3 months ${ }^{44}$. The second trial attempts to target the emergent adverse events up to the end of a maintenance treatment period of 2 years and 4 months ${ }^{45}$.

\section{PF-06650833 (PFIZER) IRAK4 INHIBITOR}

The IL-1 receptor-associated kinase (IRAK) family mediates activating signals from TLRs and IL-1 receptor ${ }^{46}$.

The IRAK family, comprised of four members, IRAK1, IRAK2, IRAK-M, and IRAK4, is closely involved in the pathogenesis of inflammatory autoimmune disorders ${ }^{46}$. The overexpression of IRAK4 encourages the generation of a pro-inflammatory environment, with increased production of IL-1 $\beta$ and tissue factor ${ }^{46}$. This explains the alleged involvement of the IRAK family in the complex pathogenesis of $\mathrm{HS}^{38}$.

A current Phase 2 multicenter study is evaluating the safety and efficacy of three kinase inhibitors (PF 06650833, PF 06700841, and PF 06826647) controlled with placebo in 192 randomized patients with moderate-to-severe HS. A primary outcome providing the percentage of patients with HiSCR is still pending ${ }^{47}$.

\section{Iscalimab (Novartis) CD40 inhibitor}

Iscalimab (CFZ533) is a fully human monoclonal antibody that blocks CD154 from binding to CD40 and therefore prevents CD40 pathway signaling and activation of CD40+ cell types ${ }^{35,48}$.

A Phase 2 study to assess preliminary efficacy and safety of CFZ533 and LYS006 in moderate-to-severe $\mathrm{HS}$ is in the recruitment period ${ }^{49}$.

\section{LYS006 (Novartis) LTA4 hydrolase inhibitor}

LYS006 is a highly potent and selective LTA4H inhibitor with high whole blood potency and long-lasting pharmacodynamic effects ${ }^{50}$.

Leukotriene A4 hydrolase is a cytosolic metalloenzyme involved in the biosynthesis of pro-inflammatory leukotriene B4 (LTB4). Furthermore, LTB4 is a strong inflammatory mediator engaged in innate and adaptative immune responses that has an important role in the recruitment and activation of neutrophils ${ }^{50}$.

CFZ533 and LYS006 are currently being investigated in the same phase 2 clinical trial in $\mathrm{HS}^{49}$.

\section{Brodalumab (Leo Pharma), IL-17 Receptor inhibitor}

The IL-17 cytokine family consists of 6 cytokines (IL-17A to F) with five different receptor subtypes (IL-17RA to RE). 
On ligation of ligand and receptor, tissue-specific transcription of genes for a host of different pro-inflammatory cytokines, chemokines, and matrix metalloproteases is initiated. In addition, IL-17 exerts its greatest inflammatory potential through its recruitment of immune cells such as TNF, IL-1 $\beta$, IFN $\gamma$, and IL-23 ${ }^{51}$.

Increase in gene expression of IL-17 in lesional skin of $\mathrm{HS}$ patients has been shown to compare with healthy skin $^{18}$.

Brodalumab is a human monoclonal antibody binding to IL-17RA and thereby enables blockade of IL-17A, IL-17C and IL-17F. This drug was approved in 2019 for moderate-to-severe psoriasis treatment. (AMAGINE-2, ClinicalTrials.gov identifier: NCT01708603. AMAGINE-3, ClinicalTrials.gov identifier: NCT01708629).

A cohort study was performed in ten participants with moderate HS to assess the safety and clinical response. Brodalumab $210 \mathrm{mg} / 1.5 \mathrm{~mL}$ was administered subcutaneously at weeks 0,1 and 2 and every two weeks thereafter until week 24 . No grade 2 or 3 adverse events were reported. All patients achieved HiSCR. Achievement occurred as early as week $2^{52}$.

In another cohort study, brodalumab $210 \mathrm{mg} / 1.5 \mathrm{~mL}$ was administered every week to a sample of ten patients with severe HS to provide better control for draining tunnels. All of the patients achieved HiSCR by week 4 sustained through week 24 , without reporting serious adverse events ${ }^{53}$.

At present, brodalumab is being studied in a Phase II clinical trial with ongoing patient recruitment, and no data from this study are yet available.

\section{P19 blockers}

$\mathrm{IL} 23$ is a pro-inflammatory member of the IL-12 cytokine superfamily with a potent ability to enhance the production of Th17 cells. IL-23 is mainly secreted by dendritic cells and activated macrophages in peripheral tissues such as the skin. IL-23 and IL-17 form an axis through Th17 cells with a strong association to activation and pathogeny of the immune system ${ }^{54}$.

Two options, guselkumab and risankizumab, are currently under Phase II clinical assays.

\section{+Guselkumab (Janssen)}

Guselkumab is a fully human $\lg \mathrm{G} 1-\lambda$ monoclonal antibody that binds to the p19 subunit of IL-23 and inhibits the intracellular and downstream signaling of IL-23. It is currently approved for psoriasis and psoriatic arthritis.
Case series following the psoriasis regimen have reported significant improvement in patients with severe $\mathrm{HS}^{55,56}$.

A Phase II trial (NCT03628924) evaluating efficacy and safety has recently been completed with a primary endpoint of measuring the percentage of participants achieving HiSCR at Week 16. Some of the published results are displayed in Fig. 1 showing discrete results in achieving complete disease control ${ }^{57}$.

\section{+RISANKIZUMab (AbBVIE)}

Risankizumab is a humanized IgG1 monoclonal antibody targeting the p19 subunit of IL-23. It is currently approved for psoriasis and psoriatic arthritis.

So far, only retrospective data on the efficacy of risankizumab within small groups of patients with HS have been reported with acceptable results ${ }^{58}$.

A Phase II (NCT03926169) trial evaluating efficacy and safety has recently been completed with results pending publication.

\section{Janus Kinase (JAK) inhibitors}

Kinases in the JAK family include JAK1, JAK2, JAK3 and non-receptor tyrosine-protein kinase TYK2 and are involved in signaling pathways affecting hematopoiesis, immunity and inflammation.

After JAKs are activated by diverse cytokines, signal transducers and activators of transcription (STATS) proteins are activated. STATS can then enter the nucleus and induce inflammation ${ }^{59}$.

\section{+BrepocitinIB (PFIZer)}

Brepocitinib is an orally available, selective inhibitor of non-receptor tyrosine-protein kinase tyrosine kinase 2 (TYK2) and tyrosine-protein kinase JAK1 (JAK1) with potential immunomodulatory and anti-inflammatory activities. On oral administration, brepocitinib selectively binds to and inhibits the activation of TYK2 and JAK1, thereby disrupting TYK2 and JAK-1-dependent cytokine signaling. This may reduce inflammatory responses and prevent inflammation-induced damage caused by certain immunological diseases.

According to Pfizer website information, brepocitinib has shown in vitro ability to inhibit IL-23, IL-12 and IFNalpha. For this reason, it is being studied for use in HS patients ${ }^{60}$.

Efficacy and safety are also being evaluated for Crohn's disease, ulcerative colitis, psoriasis, psoriatic 
arthritis, atopic dermatitis, alopecia areata, non-segmental vitiligo, and lupus erythematosus.

\section{+INCB054707 (Incyte)}

INCB054707 is a JAK1 inhibitor and has been tested for HS in two Phase II trials (NCT03569371 NCT03607487). Safety and tolerability assessment were the primary endpoints of the trial.

A safety review was conducted at week 4 , with a 30-day safety follow-up after the 8-week trial. A total of 35 participants were randomized to orally once daily of either placebo, $30 \mathrm{mg}, 60 \mathrm{mg}$, or $90 \mathrm{mg}$ doses of the drug. In the arm taking INCB054707 at $90 \mathrm{mg}$ over an 8-week period, $87.5 \%$ of the patients achieved HiSCR ${ }^{61}$.

\section{+Upadacitinib (AbbVie)}

Upadacitinib is a JAK1 inhibitor currently approved for atopic dermatitis. A clinical trial is now active but not recruiting to evaluate efficacy in HS.

Other studies are also taking place for non-segmental vitiligo, lupus erythematosus, axial spondylitis, juvenile idiopathic arthritis, rheumatoid arthritis, Takayasu arteritis, giant cell arteritis, ulcerating colitis, and Crohn's disease.

\section{+Ropsacitinib (Pfizer)}

Ropsacitinib (PF-06826647) is claimed as a TYK2 Inhibitor. However, its activity at clinically relevant concentrations to achieve TYK2 inhibition is suggestive of significant inhibition of at least JAK2, if not also of JAK1. Ropsacitinib is an ATP-competitive inhibitor.

NCT04092452 is a study with 3 kinase inhibitors (PF 06650833, PF 06700841, and PF 06826647) in participants with moderate-to-severe HS. The primary endpoint is the percentage of participants achieving HiSCR at week 16. Results are not yet published.

Phase I studies for plaque psoriasis showed significant improvement in disease activity within 4 weeks of dosing with an acceptable safety profile ${ }^{62}$.

\section{Phase III drugs: IL-17 blockers Secukinumab (Novartis)}

Secukinumab is an IL-17A inhibitor that is FDAapproved for moderate-to-severe plaque psoriasis, psoriatic arthritis, and ankylosing spondylitis. Because the expression of IL-17 gene is increased in lesions and in perilesional skin, and IL-17 serum concentration in patients with HS is significantly increased, its blockade of the IL-17 pathway could be a potential target to treat $\mathrm{HS}^{63}$.

Nine patients were enrolled and four completed an open-label pilot trial of secukinumab. Of the nine, six achieved HiSCR at week 24. Another retrospective study on a cohort of patients treated with secukinumab for their HS revealed a decrease in the median Sartorius score, the median inflammatory lesion count, and median DLQI score ${ }^{64}$, and $75 \%$ of patients (15/20) achieved a successful HiSCR at week $16^{65}$. There are two randomized double-blind multicenter trials (SUNRISE and SUNSHINE) currently being conducted of patients with moderate-to-severe HS. Their goal is to compare the efficacy, safety, and tolerability of secukinumab doses of $300 \mathrm{mg}$ dosed 2-weekly and 4-weekly ${ }^{66,67}$.

Dosing of secukinumab for patients with HS is $300 \mathrm{mg}$ administered subcutaneously weekly for 1 month, then once every 4 weeks ${ }^{68,69}$. A retrospective study showed two HS patients with no personal or family history of inflammatory bowel disease (IBD) developed Crohn's disease (CD) after 3 and 5 months of treatment respectively ${ }^{20}$, but the open-label pilot trial showed no new-onset inflammatory bowel disease or other serious adverse events ${ }^{65}$. Still, HS is associated with IBD with a pooled OR for Crohn's disease $(C D)$ and 1.5 for ulcerative colitis $(U C)^{70}$.

\section{Bimekizumab (UCB Pharma)}

Bimekizumab is a humanized, full-length IgG monoclonal antibody that selectively inhibits both IL-17A and IL-17F and which has demonstrated rapid and significant improvements in dermatologic and rheumatologic disease activity ${ }^{65}$. It is a dual-cytokine blockade that may profoundly affect chronic tissue inflammation and confer additional efficacy in immune-mediated diseases such as $\mathrm{HS}^{71}$.

Seventy-nine HS patients enrolled in a phase 2, double-blind, placebo-controlled randomized clinical trial that demonstrated that $46 \%$ of bimekizumab-treated participants achieved HiSCR75. Another 32\% achieved HiSCR90 at week 12. In the other arm of adalimumab, $35 \%$ achieved HiSCR75 and 15\% achieved HiSCR90 while $10 \%$ of placebo-treated participants achieved HiSCR75 and none achieved HiSCR90 ${ }^{26}$. Bimekizumabtreated participants reported greater improvements in skin pain at week 12 and no impact of disease on their quality of life compared with placebo-treated 
participants. Improvements in IHS4, skin pain, and DLQI were numerically similar or smaller in adalimumab-treated participants than in those treated with bimekizumab $^{65}$. Dosing is $320 \mathrm{mg}$ every 2 weeks (after a $640-\mathrm{mg}$ loading dose at baseline) ${ }^{65}$. Most adverse effects were mild or moderate, though hospitalization for anemia and empyema did occur for one patient ${ }^{71}$.

\section{Discussion}

HS is a condition that involves several concomitant pathways. Although its pathogenesis remains unclear, more studies are being conducted to identify the triggers for this debilitating disease.

Although there are no current treatments that adequately control HS, immunomodulatory treatments targeting the Th17 pathway and the JAK/STAT pathways are now being explored. Anti-IL-17 drugs such as secukinumab, bimekizumab, brodalumab, and anti-IL-23 drugs such as risankizumab or guselkumab may represent possible treatments in the near future.

Preliminary data from ongoing clinical trials suggests that IL-17 blockers are the most promising options for $\mathrm{HS}$ management. Close safety monitoring to determine the risk of secondary IBD is necessary before considering these options beneficial to achieving an integral control of HS patients.

\section{Funding}

None.

\section{Conflicts of interest}

Dr Martorell has received honorariums from sponsored symposiums and advisory board meetings and has participated as principal investigator of Phases II, III, and IV from the following companies: AbbVie, Celgene, Janssen, Novartis, Merck Sharp \& Dohme, UCB, Pfizer, Gebro Pharma, Leo Pharma, Lilly, Sandoz, Galderma. There are no conflicts of interest with respect to the research, authorship and/or publication of this article. Dr Jfri, Dr Ochando and Dr Mayo declare no conflicts of interest with respect to the research, authorship and/or publication of this article.

\section{Ethical disclosures}

Protection of human and animal subjects. The authors declare that no experiments were performed on humans or animals for this study.
Confidentiality of data. The authors declare that no patient data appear in this article.

Right to privacy and informed consent. The authors declare that no patient data appear in this article.

\section{References}

1. Goldburg SR, Strober BE, Payette MJ. Hidradenitis suppurativa: epidemiology, clinical presentation, and pathogenesis. J Am Acad Dermatol. 2020:82:1045-58.

2. Chiricozzi A, Giovanardi G, Caro DR, lannone M, Garcovich S, Dini V, et al. Alexithymia affects patients with hidradenitis suppurativa. Eur $J$ Dermatol. 2018;28:482-7.

3. Martorell A, Jfri A, Koster SBL, Gomez-Palencia P, Solera M, Alfaro-Rubio $A$, et al. Defining hidradenitis suppurativa phenotypes based on the elementary lesion pattern: results of a prospective study. J Eur Acad Dermatol Venereol. 2020;34:1309-18.

4. Martorell A, Caballero A, González Lama Y, Jiménez-Gallo D, Lázaro Serrano M, Miranda J, et al. Management of patients with hidradenitis suppurativa. Actas Dermosifiliogr. 2016;107 Suppl 2:32-42.

5. Overview of targets in development. https://clinicaltrials.gov/ct2/results?cond=hidradenitis+Suppurativa [Last accessed August 2021].

6. Kim ES, Garnock-Jones KP, Keam SJ. Adalimumab: a review in hidradenitis suppurativa. Am J Clin Dermatol. 2016;17:545-52.

7. Kimball AB, Okun MM, Williams DA, Gottlieb AB, Papp KA, Zouboulis CC, et al. Two phase 3 trials of adalimumab for hidradenitis suppurativa. N Engl J Med. 2016;375:422-34.

8. Marzano AV, Genovese G, Casazza G, Moltrasio C, Dapavo P, Micali G, et al. Evidence for a 'window of opportunity' in hidradenitis suppurativa treated with adalimumab: a retrospective, real-life multicentre cohort study. Br J Dermatol. 2021;184:133-40.

9. Zouboulis CC. First real-world data provide evidence for a 'window of opportunity' in treatment of hidradenitis suppurativa/acne inversa. $\mathrm{Br} \mathrm{J}$ Dermatol. 2021;184:10-1.

10. Blaszczak A, Trinidad JC, Cartron AM. Adalimumab for treatment of hidradenitis suppurativa during the COVID-19 pandemic: safety considerations. J Am Acad Dermatol. 2020;83:e31.

11. Grant A, Gonzalez T, Montgomery MO, Cardenas V, Kerdel FA. Infliximab therapy for patients with moderate to severe hidradenitis suppurativa: a randomized, double-blind, placebo-controlled crossover trial. J Am Acad Dermatol. 2010;62:205-17.

12. Lim SYD, Oon HH. Systematic review of immunomodulatory therapies for hidradenitis suppurativa. Biologics. 2019;13:53-78.

13. Tegtmeyer K, Atassi G, Zhao J, Maloney NJ, Lio PA. Off-label studies on anakinra in dermatology: a review. J Dermatolog Treat. 2020;1-14.

14. Ghias MH, Johnston AD, Kutner AJ, Micheletti RG, Hosgood HD, Cohen SR. High-dose, high-frequency infliximab: a novel treatment paradigm for hidradenitis suppurativa. J Am Acad Dermatol. 2020;82:1094-101.

15. Tzanetakou V, Kanni T, Giatrakou S, Katoulis A, Papadavid E, Netea MG, et al. Safety and efficacy of anakinra in severe hidradenitis suppurativa: a randomized clinical trial. JAMA Dermatol. 2016;152:52-9.

16. Leslie KS, Tripathi SV, Nguyen TV, Pauli M, Rosenblum MD. An open-label study of anakinra for the treatment of moderate to severe hidradenitis suppurativa. J Am Acad Dermatol. 2014;70:243-51.

17. Friedman BE, English JC $3^{\text {rd }}$. Drug-induced sarcoidosis in a patient treated with an interleukin-1 receptor antagonist for hidradenitis suppurativa. JAAD Case Rep. 2018;6:543-5.

18. Schlapbach C, Hänni T, Yawalkar N, Hunger RE. Expression of the IL-23/ Th17 pathway in lesions of hidradenitis suppurativa. J Am Acad Dermatol. 2011;65:790-8.

19. Montero-VilchezT,Pozo-RománT Sánchez-VeliciaL Vega-GutiérrezJ,Arias-Santiago S, Molina-Leyva A. Ustekinumab in the treatment of patients with hidradenitis suppurativa: multicenter case series and systematic review. J Dermatolog Treat. 2020;1-6

20. Romaní J, Vilarrasa E, Martorell A, Fuertes I, Ciudad C, Molina-Leyva A Ustekinumab with intravenous infusion: results in hidradenitis suppurativa. Dermatology. 2020;236:21-4.

21. Eigler A, Siegmund B, Emmerich U, Baumann K, Hartmann G, Endres S. Anti-inflammatory activities of cAMP-elevating agents: enhancement of $\mathrm{IL}-10$ synthesis and concurrent suppression of TNF production. J Leukoc Biol. 1998;63:101-7.

22. Vossen A, van Doorn M, van der Zee HH, Prens EP. Apremilast for moderate hidradenitis suppurativa: results of a randomized controlled trial. J Am Acad Dermatol. 2019;80:80-8.

23. Kerdel FR, Azevedo FA, Kerdel DC, Don FA, Fabbrocini G, Kerdel FA Apremilast for the treatment of mild-to-moderate hidradenitis suppurativa in a prospective, open-label, phase 2 study. J Drugs Dermatol. 2019;18:170-6. 
24. Weber P, Seyed Jafari SM, Yawalkar N, Hunger RE. Apremilast in the treatment of moderate to severe hidradenitis suppurativa: a case series of 9 patients. J Am Acad Dermatol. 2017;76:1189-91.

25. Jovčevska I, Muyldermans S. The therapeutic potential of nanobodies. BioDrugs. 2020;34:11-26.

26. Papp K, Weinberg M, Morris A, Reich K. IL17A/F nanobody sonelokimab in patients with plaque psoriasis: a multicentre, randomised, placebo-controlled, phase 2b study. Lancet. 2021;397:1564-75.

27. Zouboulis CC, da Costa AN, Makrantonaki E, Hou XX, Almansouri D, Dudley JT, et al. Alterations in innate immunity and epithelial cell differentiation are the molecular pillars of hidradenitis suppurativa. J Eur Acad Dermatol Venereol. 2020;34:846-61.

28. Boyles JS, Beidler CB, Strifler BA, Girard DS, Druzina Z, Durbin JD, et al. Discovery and characterization of a neutralizing pan-ELR+CXC chemokine monoclonal antibody. MAbs. 2020;12:1831880.

29. Available from: https://www.clinicaltrials.gov/ct2/show/nct04493502?ter$m=l y 3041658 \&$ cond=hidradenitis+suppurativa\&draw $=2 \&$ rank $=1$

30. Bekker P, Dairaghi D, Seitz L, Leleti M, Wang Y, Ertl L. Characterization of pharmacologic and pharmacokinetic properties of CCX168, a poten and selective orally administered complement 5 a receptor inhibitor, based on preclinical evaluation and randomized phase 1 clinical study. PLoS One. 2016;11:e0164646.

31. Kanni T, Zenker O, Habel M, Riedemann N, Giamarellos-Bourboulis EJ. Complement activation in hidradenitis suppurativa: a new pathway of pathogenesis? Br J Dermatol. 2018;179:413-9.

32. Ghias MH, Hyde MJ, Tomalin LE, Morgan BP, Alavi A, Lowes MA, et al. Role of the complement pathway in inflammatory skin diseases: a focus on hidradenitis suppurativa. J Invest Dermatol. 2020;140:531-6.e1.

33. Available from: https://www.clinicaltrials.gov/ct2/show/nct03852472?ter$m=$ avacopan $+\% 28$ chemocentry $\% 29$ \&cond=hidradenitis+suppurativa\&draw $=2 \&$ rank $=1$

34. Global Newswire. ChemoCentryx Announces Positive Topline Results of Phase II AURORA Clinical Trial of Avacopan in the Treatment of Hidradenitis Suppurativa (HS); 2020.

35. Del Duca E, Morelli P, Bennardo L, di Raimondo C, Nisticò SP. Cytokine pathways and investigational target therapies in hidradenitis suppurativa. Int J Mol Sci. 2020;21:8436.

36. Giamarellos-Bourboulis EJ, Argyropoulou M, Kanni T, Spyridopoulos T, Otto I, Zenker O, et al. Clinical efficacy of complement C5a inhibition by IFX-1 in hidradenitis suppurativa: an open-label single-arm trial in patients not eligible for adalimumab. Br J Dermatol. 2020;183:176-8.

37. Available form: https://www.clinicaltrials.gov/ct2/show/results/nc t03487276?term=ifx-1\&cond=hidradenitis+suppurativa\&draw $=2 \&$ rank $=1$

38. Wolk K, Join-Lambert O, Sabat R. Aetiology and pathogenesis of hidradenitis suppurativa. Br J Dermatol. 2020;183:999-1010.

39. Kanni T, Argyropoulou M, Spyridopoulos T, Pistiki A, Stecher M, DinareIlo $\mathrm{CA}$, et al. MABp1 targeting IL-1 $\alpha$ for moderate to severe hidradenitis suppurativa not eligible for adalimumab: a randomized study. J Invest Dermatol. 2018:138:795-801.

40. Kanni T, Argyropoulou M, Dinarello CA, Simard J, Giamarellos-Bourboulis EJ. MABp1 targeting interleukin-1 $\alpha$ in hidradenitis suppurativa ineligible for adalimumab treatment: results of the open-label extension period. Clin Exp Dermatol. 2021:46:162-3.

41. Available from: https://www.clinicaltrials.gov/ct2/show/NCT04988308?ter$\mathrm{m}=$ Bermekimab\&cond=Hidradenitis+Suppurativa\&draw $=2 \&$ rank $=2$

42. Gresnigt MS, van de Veerdonk FL. Biology of IL-36 cytokines and their role in disease. Semin Immunol. 2013;25:458-65.

43. Available from: https://www.clinicaltrials.gov/ct2/show/nct04856930?ter$m=$ imsidolimab\&cond=hidradenitis+suppurativa\&draw $=2 \&$ rank $=1$

44. Available from: https://www.clinicaltrials.gov/ct2/show/study/nct04876391? term=spesolimab\&cond=hidradenitis+suppurativa\&draw $=2 \&$ rank $=2$

45. Available from: https://www.clinicaltrials.gov/ct2/show/study/nct04762277? term $=$ spesolimab\&cond=hidradenitis+suppurativa\&draw $=2 \&$ rank $=1$

46. Su LC, Xu WD, Huang AF. IRAK family in inflammatory autoimmune diseases. Autoimmun Rev. 2020;19:102461.

47. Available from: https://www.clinicaltrials.gov/ct2/show/nct04092452?ter$m=p f-06650833 \&$ cond=hidradenitis+suppurativa\&draw $=2 \&$ rank $=1$

48. Espié P, He Y, Koo P, Sickert D, Dupuy C, Chokoté E, et al. First-inhuman clinical trial to assess pharmacokinetics, pharmacodynamics, safety, and tolerability of iscalimab, an anti-CD40 monoclonal antibody. Am J Transplant. 2020;20:463-73.
49. Available from: https://www.clinicaltrials.gov/ct2/show/nct03827798?ter$m=c f z 533 \&$ cond=hidradenitis+suppurativa\&draw $=2 \&$ rank $=1$

50. Markert C, Thoma G, Srinivas H, Bollbuck B, Lüönd RM, Miltz W, et al. Discovery of LYS006, a potent and highly selective inhibitor of leukotriene $A_{4}$ hydrolase. J Med Chem. 2021;64:1889-903.

51. Onishi RM, Gaffen SL. Interleukin-17 and its target genes: mechanisms of interleukin-17 function in disease. Immunology. 2010;129:311-21.

52. Frew JW, Navrazhina K, Grand D, Sullivan-Whalen M, Gilleaudeau $P$, Garcet $S$, et al. The effect of subcutaneous brodalumab on clinical disease activity in hidradenitis suppurativa: an open-label cohort study. J Am Acad Dermatol. 2020;83:1341-8.

53. Frew JW, Navrazhina K, Sullivan-Whalen M, Gilleaudeau P, Garcet S, Krueger JG. Weekly administration of brodalumab in hidradenitis suppurativa: an open-label cohort study. Br J Dermatol. 2021;184:350-2.

54. McKenzie BS, Kastelein RA, Cua DJ. Understanding the IL-23-IL-17 immune pathway. Trends Immunol. 2006;27:17-23.

55. Casseres RG, Kahn JS, Her MJ, Rosmarin D. Guselkumab in the treatment of hidradenitis suppurativa: a retrospective chart review. J Am Acad Dermatol. 2019;81:265-7.

56. Kovacs M, Podda M. Guselkumab in the treatment of severe hidradenitis suppurativa. J Eur Acad Dermatol Venereol. 2019;33:e140-1.

57. Available from: https://www.clinicaltrials.gov [Last accessed on 2021 Aug]

58. Marques E, Arenberger P, Smetanová A, Gkalpakiotis S, Zimová D, Arenbergerová $M$, et al. Successful treatment of recalcitrant hidradenitis suppurativa with risankizumab after failure of anti tumour necrosis factor alpha. Br J Dermatol. 2021;184:966-7.

59. Zarrin AA, Bao K, Lupardus $P$, Vucic D. Kinase inhibition in autoimmunity and inflammation. Nat Rev Drug Discov. 2021;20:39-63.

60. Pfizer Pipeline; 2020. Available from: https://www.pfizer.com/science/ drug-product-pipeline [Last accessed on 2020 Oct 27].

61. Alavi A. Oral Session 4b, Oral Presentation at EHSF; 2021.

62. Tehlirian C, Peeva E, Kieras E, Scaramozza M, Roberts ES, Singh RS, et al. Safety, tolerability, efficacy, pharmacokinetics, and pharmacodynamics of the oral TYK2 inhibitor PF-06826647 in participants with plaque psoriasis: a phase 1, randomised, double-blind, placebo-controlled, parallel-group study. Lancet Rheumatol. 2021;3:e204-13.

63. Kelly G, Hughes R, McGarry T, van den Born M, Adamzik K, Fitzgerald R, et al. Dysregulated cytokine expression in lesional and nonlesional skin in hidradenitis suppurativa. Br J Dermatol. 2015;173:1431-9.

64. Prussick L, Rothstein B, Joshipura D, Saraiya A, Turkowski Y, Abdat R, et al. Open-label, investigator-initiated, single-site exploratory trial evaluating secukinumab, an anti-interleukin-17A monoclonal antibody, for patients with moderate-to-severe hidradenitis suppurativa. Br J Dermatol. 2019;181:609-11.

65. Reguiaï Z, Fougerousse AC, Maccari F, Bécherel PA. Effectiveness of secukinumab in hidradenitis suppurativa: an open study (20 cases). J Eur Acad Dermatol Venereol. 2020;34:e750-1.

66. US National Library of Medicine. Study of Efficacy and Safety of Two Secukinumab dose Regimens in Subjects with Moderate to Severe Hidradenitis Suppurativa (HS), SUNRISE; 2018. Available from: https:// www.clinicaltrials.gov/ct2/show/nct03713632? cond=\%28\%22hidradenitis+suppurativa\%22+or+\%22acne+inversa\%22\%29\&rank=8 [Last accessed on 2021 Sep 24].

67. US National Library of Medicine. This is a Study of Efficacy and Safety of Two Secukinumab dose Regimens in Subjects with Moderate to Severe Hidradenitis Suppurativa (HS), SUNSHINE; 2018. Available from: https://clinicaltrials.gov/ct2/show/nct03713619?cond=\%28\%22hidradenitis+suppurativa $\% 22+$ or+\%22acne+inversa $\% 22 \% 29 \&$ rank=9 [Last accessed on 2021 Sep 24].

68. Thorlacius L, Theut Riis $P$, Jemec $G$. Severe hidradenitis suppurativa responding to treatment with secukinumab: a case report. $\mathrm{Br} \mathrm{J}$ Dermatol. 2018;179:182-5

69. Schuch A, Fischer T, Boehner A, Biedermann T, Volz T. Successful treatment of severe recalcitrant hidradenitis suppurativa with the interleukin-17A antibody secukinumab. Acta Derm Venereol. 2018;98:151-2.

70. Chen WT, Chi CC. Association of hidradenitis suppurativa with inflammatory bowel disease: a systematic review and meta-analysis. JAMA Dermatol. 2019;155:1022-7.

71. Glatt S, Jemec GB, Forman S, Sayed C, Schmieder G, Weisman J, et al. Efficacy and safety of bimekizumab in moderate to severe hidradenitis suppurativa: a phase 2, double-blind, placebo-controlled randomized clinical trial. JAMA Dermatol. 2021:e212905. 